NOTAS Y DISCUSIONES

\title{
Amando lo artificial: Ortega y Gasset y nuestra relación con la técnica hoy
}

\author{
PATRICK H. DUST \\ Carleton College Northfield, Minnesota, Estados Unidos
}

En un ensayo de 1935 intitulado "Misión del bibliotecario», José Ortega y Gasset nos ofrece una preciosa historia del "libro». Explica cómo durante siglos el libro era vivido por los seres humanos como una maravilla y una esperanza, y luego cómo, en las primeras décadas de nuestro siglo, ese artefacto comenzó a convertirse en algo ambiguo y hasta negativo. Comenta:

[...] todo la que el hombre inventa y crea para facilitarse la vida, todo eso que llamamos civilización y cultura, llega un momento en que se revuelve contra él. Precisamente porque es una creación queda ahí, en el mundo, fuera del sujeto que lo creó, goza de existencia propia, se convicrte en cosa, en mundo frente al hombre, y lanzado a su particular e inexorable destino, se desentiende de la intención con que el hombre lo cré para salir de un apuro ocasional [...] La economia, la técnica, facilidades que el hombre inventa, le han puesto hoy cerco y amenazan estrangularle [...]. Las ciencias, al engrosar fabulosamente y multiplicarse y especializarse, rebasan las capacidades de adquisición que el hombre posee y le acongojan y oprimer como plagas de la naturaleza. El estudio no es ya el otium, la schole, que fue en Grecia - empieza ya a inundar la vida del hombre y rebasar sus límites. La inversión carac- terística de esa rebelión contra su creador de las creaciones humanas es ya inminente: en vez de estudiar para vivir va a tener que vivir para estudiar.'

La inversión característica que vivimos nosotros hoy puede describirse así: en vez de poner nuestra técnica al servicio de ideales humanos, vivimos de acuerdo con las demandas que nos impone ella (la eficacia, la rapidez, la productividad máxima, etc.). En vez de inventar para vivir, nosotros vivimos ya para inventar. Ortega tenía - y sigue teniendo-razón. La técnica nos oprime como una plaga de la naturaleza.

Quizás pueda servir aquí una comparación. Cuando el animal vive en su medio ambiente, tiene que adaptarse a éste o se muere. Es capaz de introducir pequeñas modificaciones en su mundo externo, pero no puede transformarlo en sentido dramático, no puede, por ejemplo, reemplazarlo del todo con una construcción artificial. A diferencia del animal, sin embargo, el hornbre posee el poder de rehacer ese mundo, de tomar posesión de él, de convertir el universo material en instrumento con el cual fabricarse otro mundo menos pri- 
mitivo y más ahumano». Es esta una de las verdades que se desprenden de la famosa fórmula "yo soy yo y mi circunstanciar. Pero el pasaje citado también llama la atención por otra razón. Si lo observamos bien, descubrimos que Ortega no describe la técnica como lo hacen muchos otros, es decir, como una anomalidad monstruosa dentro de la economía humana -el equivalente moderno del "pecado" en tiempos anteriores-, sino más bien como una posibilidad permanente de todo ser humano en cualquier época. Al tin y al cabo, lo que sucede con la técnica no es diferente de lo que sucede, en el fondo, con el libro, con la economía, o con las ciencias y el estudio, ni de lo que puede suceder, en principio, con todas las creaciones auténticamente humanas.

Con el espacio de que dispongo en estas páginas, es imposible ofrecer un panorama detallado de las muchas tendencias e investigaciones que existen en el campo de la filosofía de la técnica en mi país. ${ }^{2}$ Por eso mi propósito aquí cs bastante limitado. Yo quisiera hacer unas observaciones sobre el tema, enfocándolas de modo que puedan incorporarse a una especie de diálogo con ideas claves que tenía Ortega sobre la técnica. Esto me permitirá sugerir algo de la reacción probable del filósofo madrileño a los nuevos acontecimientos dentro de la filosofía de la técnica en los Estados Unidos. Ahora bien, buenos ejemplos de la filosofía de la técnica norteamericana son tres libros que comento a continuación: 1) Technology and the Character of Contemporary Life: A Philosophical Inquiry por Albert Borgman (1984), 2) Electric Language: A Philosophical Sttudy of Word Processing por Michael Heim (1987) y 3) Technology and the Lifeworld: From Garden to Earth por Don thde (1990). Resumiré brevemente el asunto de cada libro, comentaré su relación con el contexto más amplio de la filosofía continental, y luego especularé sobre la reacción probable de Ortega.

Albert Borgman es muy conocido en este campo. Technology and the Character of Contemporary life representa la culminación de muchos años de meditación y de un compromiso profundamen. te personal y sólidamente intelectual. ${ }^{3}$ Desde el principio Borgman ataca la visión común y corriente de la tecnología como un instrumento neutral que puede ser dominado fácilmente por un acto de la voluntad, e intenta reemplazar esa idea con la imagen de un fenómeno que se ha confundido en forma mucho más sutil y omnipresente con todas las actividades de nuestra vida. Así, desde el principio la preocupación con cómo la tecnología afecta en maneras concretas nuestra vida cotidiana es evidente y la cuidadosa fenomenologia de esa interacción es una de las mayores virtudes del libro. El autor aísla y describe lo que llama "el paradigma del recurso» (paradigm device) para desarrollar su tema. Este paradigma se refiere al proceso en que nos amoldamos a los requisitos de la técnica y poco a poco vamos perdiendo nuestras cualidades humanas. En una época en que reina la tecnología las cosas más mínimas de la vida tienden a transformarse y perder su sentido familiar. Al mismo tiempo, hay que destacar el hecho de que Borgman evita el fatalismo diciendo que la gente está «inplicada" en este proceso, y no está dominada del todo. ¿Y cuál es la respuesta a esta implicación? Es una reforma de las actividades de nuestra vida diaria. Enfocándose sobre todo en tres ejemplos - nuesira relación estética con la naturaleza, la cultura de la mesa, y el correr-, Borgman elabora una segunda fenomenología de lo que llama "prácticas focales" con el objeto de mostrar cómo podemos volver a afirmar experiencias que aún no han sido transformadas y deshumanizadas por la tecnología. 
Nuestro segundo ejemplo, Michael Heim, estudia lo que a primera vista parece un problema más restringido que el de Borgman, nuestra creciente fascinación por los ordenadores. Pero dentro de ese contexto lo que le preocupa es un fenómeno amplio y fundamental, que es nada menos que la dramática reinvención del yo mediante la escritura digital. En su libro Electric Language," Heim desarrolla una teoría amplia sobre lo que es el alfabetismo en general $y$, empleando intuiciones de Walter Ong, Martin Heidegger y Eric Havelock, reflexiona de manera original sobre las consecuencias de nuestra fascinación por la escritura electrica. El hombre que se expresa con el ordenador, se nos dice, habita un mundo nuevo donde existe una superabundancia de posibilidades y donde la experiencia humana de la verdad tiende a desestabilizarse y ceder a otra más artificial. El resultado de todo esto es la anticipación de una utopía en la cual los símbolos se vuelven primero anónimos y luego vacíos, el perfeccionismo viene a ser omnívoro debido a la asombrosa proliferación de pseudo-posibilidades, todo es supeditado a las demandas de la realidad artificial, y se rinde culto al idolo de un control absoluto y total. El resultado es lo que Heim llama sel mundo integrado del Dominio Total» - «the integral world of Total Management"- (p. 203).

En esa extraña entrecara con el ordenador, ¿qué le pasará al hombre? En esa zona casi virtual donde todo es manipulado, procesado y controlado al máximo, ¿no dejará de existir? Según Heim, el hombre sí desaparecerá, a menos que inventemos maneras concretas de sobrevivir nuestra invención, maneras de romper el hechizo inducido por la máquina, y de reafirmar nuestra espontaneidad en el mismísimo acto de escribir. Así describe varias cosas que podemos hacer: técnicas orientales de esoltar» (re- leasing), un procedimiento que llama blockbusting - la reduccion del texto en la pantalla a múltiples bloques que mantienen las ideas por más tiempo fuera de la clausura digital-, y el cultivo de un nuevo tipo de escritura llamado «método natural», en la que la exploración abierta y no lineal de intuiciones espontáneas internumpe la ordenación exclusivamente lógica que caracteriza nuestro escribir eléctrico. Todas estas soluciones presuponen la conquista de una nueva autodisciplina. Son lo que Heim llama "actividades regresivas" cuyo propósito es ayudarnos a volver a tomar cierto control sobre un fenómeno que amenaza rehacemos a su propia imagen. Pero también vale la pena insistir en que Heim no es ningún ludista alarmista que predicara la radical destrucción de toda la tecnología. El autor sabe, lo mismo que Borgman antes que él, que la modernidad no puede ser borrada asi no más, simplemente porque no nos gustan algunas de sus consecuencias. En fin, Heim reconoce que la solución consiste en aceptar la presencia de la técnica y en fomentar una interacción más cautelosa y más sana con ella que restableciera el equilibrio natural entre el hombre y sus invenciones.

Don Thde es el filósofo que servirá como el último representante de esta nueva disciplina. Comenzó a publicar en este campo cuando este apenas existía, y dedicó varios estudios al tema, pero sin duda su tratamiento más ambicioso de la técnica aparece en su $\mathrm{Te}$ chnology and the Lifeworld: From Garden to Earth (1990), sa alusión al Lebenswelt en el título sugiere la preparación de Ihde en la fenomenologia, y es muy evidente su deuda con el pensamiento de Husserl, Merleau-Ponty y Heidegger. Reconoce que la misma textura de nuestra realidad contemporánea es profundamente tecnológica. Ihde quiere reformular sistemáticamente el 
fenómeno y explorar una nueva serie de preguntas, relacionándolas con un contexto que es decididamente material e histónico. Así su libro ofrece una tipologia de tres experiencias diferentes de la técnica, dando un análisis bastante detallado de cada una. A la primera experiencia Ihde la llama «encarnadas. Por ejemplo cuando uno lleva gafas o usa un telescopio entra en una situación que cambia la relación natural con el mundo. El aparato corrige un error o trasciende un límite y nos permite establecer un contacto más fiel con nuestro entorno material. No trata de transformar lo que es la realidad sino hacer que ésta sea más transparente de lo que seria sin el artefacto. La segunda experiencia es la "hermenéutica", como por ejemplo cuando empleamos un mapa o algún instrumento científico que produce una abstracción de lo real. Aquí una construcción semiótica media entre nosotros y las posibilidades que todavía se relacionan con el universo material. Un pianista produce sonidos en el aire que a su vez constituye otro mundo, pero un mundo que todavia es inseparable del primer mundo, del juego entre las vibraciones del aire y el oído humano. Aquí uno se sirve de algo - mapa, simulación de ordenador, piano-mientras recuerda que el acto de la interpretación pertenece al sujeto, que sigue más - menos bajo su control. A la tercera experiencia de la técnica Thde la llama "de alteridad", es decir, el instrumento emerge ahora como otro sujeto que, cara a cara con el hombre, se afirma en su poderosa artificialidad. La inteligencia artificial es un buen ejemplo, 0 , diría yo, las máquinas de realidad virtual. $\mathrm{Si}$ en las relaciones hermenéuticas la tecnología sólo empieza a emerger como entidad independiente, en las relaciones de alteridad da un paso grande hacia delante y amenaza disminuir y desplazar al hombre. Según Ihde las tres experiencias de la técnica forman un continuo en el que la percepción y la interpretación a menudo se confunden y donde importa mucho la preocupación por una praxis todavia humana.

A diferencia de Borgman y Heim, Ihde no da tanto énfasis en su análisis a la dimensión negativa de la tecnología. Es porque él encuentra una esperanza en el hecho de que nuestro mundo esté experimentando una explosión del conocimiento que, comparable quizás en sus efectos a la revolución copernicana o a la darwiniana, puede transformarnos en un sentido positivo. Este movimiento rápido hacia una civilización que él designa "pluricultural» y umultiestable» hace que nuestra relación con la técnica sea ambigua y no completamente determinada. Así dice Ihde que «las predicciones catastróficas sobre la uniformidad analítica de Marcuse, de la victoria total de la técnica de Jacques Ellul, y de la tiranía del pensar calculador de Heidegger son enróneas» (p. 159). En ultima instancia las tecnologías inclinan pero no determinan, y es probable que la acentuada diversidad cultural que vemos hoy por todas partes - una consecuencia del desarrollo de las técnicas de comunicación- haga que nuestra situación no sea tan desastrosa como muchos temen. Ihde no es excesivamente optimista - reconoce que existe mucho peligro- pero es obvio que no quiere repetir el lugar común de que la técnica está destruyendo nuestra civilización. En el último capítulo Ihde dice que este libro no es más que un estudio preliminar y termina llamándonos la atención sobre la importancia decisiva de una ética global que conserve los recursos naturales y la necesidad de edesmasculinizar" la ciencia tecnológica.

Ahora bien, ecómo habria reaccionado Ortega ante las investigaciones de estos tres filosofos? ¿Habría estado de acuerdo con los cuadros generales que 
presentan y las conclusiones a que llegan? Creo que tenemos que responder que "sí" y que "no" a estas preguntas. Primero varnos a examinar las ideas en que coinciden. En Meditación de la técnica (1933) y en el ensayo "El hombre más allá de la técnican (1951) Ortega también reconoce que la tecnología moderna ha dejado de ser neutral, y que la verdad es que cambia radicalmente la estructura de la vida humana. Dice que nuestra época es la primera en la historia humana en que el hombre en su papel de ingeniero amenaza suplantar al hombre como soñador. En una situación normal, la técnica está puesta al servicio de un programa vital que el hombre previamente se ha imaginado. Pero en el siglo XX somos testigos de los comienzos de una inversión monumental de esta situación. Y el programa de vida, que hasta ahora era el que mandaba, parece sucumbir y se pone al servicio de la capacidad técnica en sí. Con su acostumbrada exactitud, Ortega lo explica de la manera siguiente:

[...] la técnica, al aparecer por un lado como capacidad, en principio ilimitada, hace que el hombre, puesto a vivir de fe en la técnica y sólo en ella, se le vacíe la vida. Porque ser técnico y sólo técnico es poder serlo todo y consecuentemente no ser nada determinado. De puro llena de posibilidades, la técnica es mera forma hueca - como la lógica más formalista-; es incapaz de determinar el contenido de la vida. Por eso estos años en que vivimos, los más intensamente técnicos que ha habido en la historia humana, son de los más vacios $[t, 5, \mathrm{p}, 366]$.

Estas ideas no desentonan con las críticas de Borgman y Heim ni con las perspectivas fenomenológicas de Thde. En el punto de partida de sus libros respectivos se encuentra una conciencia semejante de los peligros que vienen con la técnica moderna. Y por su parte, ellos también adoptan una actitud que insiste, igual que Ortega, en que ha llegado el momento de distanciarnos del fenómeno y tomar responsabilidad por el. Creo que Ortega aceptaría la dirección general de estos estudios: le gustaría la descripción de la técnica "implicada" en las actividades cotidianas, aplaudiría la sabia inserción del ordenador en la problemática más amplia (y más humana) del alfabetismo, y aceptaría las descripciones de la relación entre las variadas experiencias de la técnica y nuestro mundo vital.

Al mismo tiempo, como dije antes, hay conclusiones en estos trabajos que Ortega jamás aceptaría. Para comprender estas diferencias, hace falta examinar primero otro problema. Cada uno de los investigadores norteamericanos -como ellos mismos reconocen - tiene una deuda grande con el pensador que muchos consideran el filósofo más importante de nuestro siglo. Me refiero a Martin Heidegger. En cada uno de los planteamientos que hemos presentado, el pensamiento del prodigioso alemán actúa como una presencia decisiva. Por eso no podemos ignorar del todo la índole y la extensión de esa influencia, ni los esfuerzos que hace cada uno por librarse de ella.

Pero veamos la obra de Heidegger. Hay una profunda división entre el primer Heidegger, autor de Ser y tiempo, y el Heidegger tardío que abandona el discurso normal y cultiva un pensar esencialmente poético. La elaboración del Dasein como entrada al problema del Ser y el proyecto de crear una nueva ontología dentro del ámbito de la filosofia constituyen las preocupaciones centrales de Ser y tiempo; mientras que una negación de toda afilosofía", y una violenta retirada de la voluntad de poder y de la técnica como manifestación epocal de esa actitud -que es nada menos que la umodernidad" - forman el núcleo de la obra tardía. El autor de estas obras tardías concibe la lengua como 
un vehículo cuasi divino que pone al hombre en contacto con algo anterior a la distinción platónica entre el sujeto y el objeto, con algo primordial que ha sido olvidado, y con el cual el hombre tiene que volver a relacionarse. Dentro de ese esquema el fin del pensar metafísico coincide con la culminación de la técnica modema.

Es importante hacer constar que en las dos trayectorias hay en el fondo una orientación profundamente nostálgica. Es decir, cara a cara con la encrucijada que define la situación humana, Heidegger opta por seguir una dirección en vez de otra. Esa encrucijada es el momento en que el hombre - sea éste concebido como animal racional, cosa pensante, conciencia husserliana, pregunta por el Ser, ente histórico, como se quiera- descubre que haga lo que haga no puede eliminar la distancia que lo separa de lo que no es él (la naturaleza, los dioses, Dios, el Ser, o lo que sea). Es decir, tarde o temprano el filósofo auténtico descubre el hecho de una diferencia, de un abismo insalvable que existe entre él y el resto del universo. ¡He aquí un momento peligroso para todo filosofo! Ante este pavoroso descubrimiento hay dos respuestas o reacciones que son posibles: o uno puede volver rápidamente la vista atrás y buscar un medio para recobrar su ser íntegro, insistiendo en que es posible reparar esa ruptura y reunirse con algo que en nuestros oscuros origenes preexistía la separación; o, por otro lado, uno puede tolerar esa visión de la diferencia - lo cual no es fácil-, tratar de aceptarla, y dedicarse a la tarea de convertirla en algo positivo. En otras palabras, o uno puede entregarse a una nostalgia infinitamente atractiva, o puede renunciar a esta, aceptando la imposibilidad de eliminar esa ruptura, y puede lanzarse a la aventura de mejorar esta vida tal cual es.

Repito, en las dos trayectorias de Heidegger, hay una orientación profunda- mente nostálgica. Era ésta esencialmente impura en Ser y tiempo porque coexistía difícilmente con el tan debatido existencialismo del autor, y también con lo que hoy vamos viendo era su no menos importante pragmatismo. ${ }^{6}$ Estas dos filosofías - la primera en su versión atea y la segunda en total- son buenos ejemplos de la segunda reacción a la encrucijada existencial que acabo de describir; son manifestaciones de una negación de la nostalgia. En los escritos del segundo Heidegger, por otro lado, la orientación conservadora deja de contaminarse con residuos subjetivistas; se intensifica y sobre todo se depura hasta tal punto que puede decirse que culmina en esa su famosa declaración en la entrevista con Der Spiegel de que "sólo puede salvarnos un dios". 'A Ahora bien, Michael Yeim y Albert Borgman se inspiran sobre todo en este Heidegger tardío, mientras que Don Ihde debe más al autor de Ser y tiempo. Hay, por supuesto, alusiones a ideas de ambos Heidegger en los tres estudios, pero cuando uno examina la dirección fundamental de cada uno de los proyectos, se descubre el predominio ya indicado.

Veamos primero el caso de Heirn. Ia huella del segundo Heidegger se ve muy clara cuando él caracteriza las únicas opciones que son posibles en nuestro mundo hoy como dos: o hacemos algo ahora para protegernos de las consecuencias del escribir digital o seremos devorados por "el mundo integral del Dominio Total». Esta misma frase «the integral world of Total Management» es, como confiesa el autor, una versión modernizada de lo que el alemán llamaba Gestell ("encuadramienton) y que se refiere a una obsesión con una productividad sin límites que no es compatible con nuestras raíces en algo humano, es decir, en algo anterior a la técnica. $Y$ ¿cómo define Heirn «lo humano"? Al modo característicamente heideggeriano como una capa- 
cidad de vivir en y desde la lengua, es decir, desde la metáfora. En Electric Writing, el hombre sólo es auténticamente humano cuando es poético, es decir, cuando está viviendo desde metáforas primordiales. Los ordenadores, con su modo informacional deshumanizado amenazan destruir al hombre en este sentido. Por eso cuando Heim comenta el trabajo de Borgman, dice que éste no ha profundizado lo suficiente en la dimensión ontologica de la técnica:

[...] las präcticas focales no nos permiten mantener la integridad psíquica dentro de la entrecara misma. Esas prácticas [son] patrones que tienen que ejercerse dentro del elemento electrónico mismo. Para descubrir el verdadero impacto de la perspectiva ontológica, debemos orientamos al impulso originante del mundo mismo, es decir, el lugar donde las cosas vienen a la presencia y en el que la psique puede realizarse plenamente [p. 233].

Toda la teoria del alfabetismo que elabora Heim tiene como base esta nostalgia, y tiene como propósito la tarea de conservar al hombre como ente metafórico cuyo destino es relacionarse con el Ser. Las actividades que Heim llama "regresivas" y que defiende al final de su libro son puramente heideggerianas. Por eso, cuando nos dice que él ha aceptado la modernidad y que nuestro problema hoy consiste en aprender a vivir con la técnica, no debemos tomarlo al pie de la letra. Vivir con la técnica significa para él no dejar que el hombre cambie, sino sólo dejar que cambien las maneras en que se relaciona con el Ser.

El estudio de Borgman es un poco más complicado. Los especialistas que han reseñado Technology and the Character of Contemporary Life han aludido a la fuerte influencia de Heidegger alli. Borgman reconoce que hay una deuda con el filósofo alemán y por eso hace hincapié en las diferencias que los separan y que contribuyen a la originalidad de su perspectiva. Así, por ejemplo, critica a Heidegger por los problemas sin solución que su historia de la metafísica plantea, y por su visión aparentemente determinista del fenómeno. $\mathrm{E}$ insiste en que es necesario ir más alla de Heidegger en dos aspectos fundamentales. Primero, en vez de buscar soluciones en ámbitos pretecnológicos, en un mundo raro que existía antes de Platón y de la modernidad, Borgman insiste en que podemos dirigirnos a las cosas en forma humana en nuestro mundo de aqui y ahora. El cuidado con que preparamos una comida, la apreciación de la hermosura de la naturaleza, la conciencia que acompaña el correr son prácticas actuales que resisten el avance de la técnica y se destacan precisamente contra el aparente dominio de su adversario. La segunda manera en que Borgman se separa de Heidegger tiene que ver con la importancia que debe tener el contexto social y político. Borgman sabe que, para iniciar una verdadera reforma de la técnica, hace falta tener en cuenta algo más que una misteriosa relación con el Ser; sabe que las decisiones institucionales -sobre todo, las democráticas- no dejan de ser importantes. "Lo que hay que mostrar - dice-. es que las cosas focales pueden florecer dentro de las prácticas humanas" (p. 200).

Pero, $\dot{c}$ de verdad ha logrado Borgman alejarse de la influencia de Heidegger? ¿Comprende que ese apego del alemán a algo que preexiste al hombre y que es superior a él -porque es la antítesis de la voluntad de poder- es la expresión de una nostalgia ante esa encrucijada existencial que describimos antes? En fin, ¿sabe Borgman cuán difícil es tolerar esa ruptura y aceptar la diferencia que el hombre irremediablemente es? Creo que la respuesta a estas preguntas es «no». Porque se encuentran en los análisis de Borgman dos constantes que corren a través de todas sus descripcio- 
nes: primero, una defensa apasionada de una relación entre el hombre y algo que no es él, una relación que reduce al hombre a una criatura cuyo destino consiste en relacionarse con ese algo indispensable; y segundo, un amor profundo y casi sin límites por esa otra realidad. Porque hay que admitir que Borgman tiene una enorme sensibilidad por las maneras no intelectuales, es decir, estéticas, en las cuales el hombre apropia las cosas de su mundo. Todas las cosas tienen una profundidad, un valor oculto que el hombre, si adopta la actitud necesaria, puede descubrir. Esta sensibilidad poética de Borgman es a la vez la gran virtud y - cuando se convierte en sentimentalismo- el gran vicio de su libro. Pero, sobre todo, es el indice más claro de su deuda con Heidegger. Muchos de los epígonos del pensador alemán son muy cerebrales en su asimilación del pensamiento del maestro. Borgman es fiel a la dimensión poética de Heidegger. Cree $-\mathrm{y}$ sus muchas descripciones de las prácticas focales lo muestran - que el hombre es más humano cuando se entrega sin rescrvas a algo que le trasciende y que es, en términos ontológicos, mucho más sólido y coherente que él. Esta misma actitud de reverencia ante la esencia de las cosas caracteriza otros escritos de Borgman también. Por ejemplo, en un artículo sobre el impacto ético de las realidades artificiales, proclama: «reality warrants itself in its epiphany", "la realidad se justifica a sí misma en su propia revelación". Y continúa: ues un singulare tantum o un nombre propio. No hay más que una realidad. $Y$ ésta lo abarca todo y es insuperable. De ahí su poder supremon. ${ }^{8} \mathrm{Y}$ el autor termina invitándonos a ser, como el, «testigos» ficles de esa realidad. Ahora la relación entre el hombre y ese mundo primordial no es sólo poética sino religiosa. Borgman confiesa ser adepto de lo que llama un "catolicismo precario», de una fe, en sus palabras, "sacramental y comunal, centrada en cosas sagradas y en comunidades vivas"." En fin, en su afirmación metafísica de estas cosas humildes, Borgman es primero heideggeriano y luego católico. El hombre tiene responsabilidad por su mundo, es libre para entregarse a la autoridad de éste o no. Heidegger se refiere a la vocación más alta del hombre como el de ser «pastor del Ser»; Borgman hace resaltar la responsabilidad del hombre libre por afirmar un mundo que no es su propia invención. En ambos es evidente que la nostalgia es el impulso más profundo. El pensar auténtico no es calculador ni tecnológico; en el fondo es poético y religioso. $\mathrm{Y}$ ahora comprendemos por qué Heim se equivoca cuando critica a Borgman por no ser suficientemente heideggeriano. Lo es tanto o más que el mismo Heim.

Como vamos viendo, no es una exageración afirmar que el subsuelo ideológico de los trabajos de Borgman y Heim es una nostalgia de raíz heideggeriana. Y podemos estar seguros de que Ortega, con su nariz perdiguera para problemas de este tipo, habría comprendido todo lo que acabamos de ver. Creo que Ortega habría tenido esto en cuenta porque su propio pensamiento, su filosofía de la razón histórica, escogió el otro camino en esa encrucijada decisiva. Es decir, optó por aceptar nuestra vida como la separación irremediable que es y decidió hacer todo lo posible por convertir esa diferencia que somos en algo positivo. En un ensayo de 1932, Ortega criticó acerbamente a otro pensador alemán extraordinario, Goethe, precisamente porque éste, después de haber descubierto la realidad radical de la vida de cada cual, huyó de su destino y se refigió en la seguridad que le ofrecfan Weimar, la poesía y la naturaleza. La vida, dice Ortega en ese ensayo, 
[...] es en si misma y siempre un naufragio. Naufragar no es ahogarse. El pobre humano, sintiendo que se sumerge en el abismo, agita los brazos para mantenerse a flote. Esa agitación de brazos [...] es la cultura [...] [Y agrega:] Goethe se acostumbró a flotar sobre la vida $\rightarrow$ se olvidó de que era un náufrago [t. 4, p. 397].

El primer Heidegger tonía una conciencia oscura de esto; el segundo huyó de esta verdad y se refugió en una ontoteología y una poesía no menos problemáticas, es decir, nostálgicas, que las de Goethe. Dije antes y conviene repetir ahora que esta nostalgia metafísica es infinitamente atractiva y dificilísima de evitar. Pero, según Ortega, el destino del hombre, no sólo de Goethe y los dos Heidegger, sino el de Borgman, Heim, Thde, de todos ustedes y el mío, si somos filosofos, es afrontar esa encrucijada y tomar una decisión. Optamos o por la nostalgia o por la aventura, y nuestra filosofia es como un espejo de esa decisión. Ortega lo explica bien por los mismos años que el ensayo sobre Goethe, en Algunas lecciones de metafisica:

[...] vivir es existir aquí y ahora; como antes he dicho nadando en el aquí y en el ahora, no es una circurstancia imaginaria. Por eso debe parecemos idiota todo lo que no sea comenzar por aceptar alegremente la circunstancia de su efectiva realidad. Ante lo fatal lo úmico con sentido que se puede hacer es aceptarlo. Eso es lo primero, luego ya veríamos si podemos en alguna medida mejorar esa circumstancia, sacarle e] mayor provecho posible $[t .12$, p. 76].

Aquí se ve también el verdadero sentido de la historicidad para Ortega y de su famosa declaración de que "el hombre no tiene una naturaleza sino una historia». Al fin y al cabo, encontrarse con una historia significa descubrir que uno es el movimiento perpetuo, el cambio inevitable; es tener una conciencia clara de que uno es una libertad, en principio, sin lírnites. Como dice Ortega en Historia como sistema:

[...] soy por fuerza libre, lo soy quiera o no. La libertad no es una actividad que ejercita un ente, el cual aparte $y$ antes de cjercitarla tiene ya un ser fijo. Ser libre quiere decir carecer de identidad constitutiva, no estar adscrito a un ser determinado, poder ser otro del que se era y no poder instalarse de una vez y para siempre en ningún ser determinado. Lo único que hay de ser fijo y estable en el ser libre es la constitutiva inestabilidad [t. 6, p. 34].

Esta es la circunstancia que uno tiene que aceptar, como dijo Ortega antes, kalegremente $\%$ es el punto de partida para la serie de proyectos que han constituido a la humanidad a través del tiempo. Cuando esa libertad que es el hombre se encuentra en el mundo, una realidad no libre sino relativamente fija y resistente, el resultado del choque de los dos es la enorme variedad de posibilidades realizadas, y por realizar, que es la historia humana.

El hombre es una entidad infinitamente plástica de la que se puede hacer lo que se quiera. Precisamente porque ella no es de suyo nada, sino mera potencia para ser "como usted quiera". Repase en un minuto el Iector todas las cosas que el hombre ha sido, es decir, que ha hecho de si - desde el usalvajew paleolítico hasta el joven surrealista de Paris. Yo no digo que en cualquier instante puede hacer de sí cualquier cosa. Eni cada instante se abren ante el posibilidades limitadas; limitadas precisamente por lo que ha sido hasta la fecha. Ésta es la única limitación concreta que el hombre tiene: su pasado. Pero si se toman, en vez de un instante, todos los instantes, no se ve qué fronteras pueden ponerse a la plasticidad humana. De la hembra paleolítica han salido Madame Pompadour y Lucila de Chateaubriand: del indigena brasileño, que no puede contar arriba de cinco, salieron Newton y Enrique Poincaré [t. 5, p. 200].

Y del pigmeo más primitivo, podemos añadir, salieron Ben Franklin, la revolución industrial, Albert Einstein, Robert 
Oppenheimer, y toda nuestra prodigiosa técnica contemporánea. $\mathrm{Si}_{\text {, }}$ parece que no hay fronteras a la plasticidad humana. Y conviene observar que en este pasaje Ortega no se está refiriendo sólo al hombre moderno de los últimos siglos. Habla del hombre desde el momento en que apareció como tal, en sentido rigurosamente metafísico. Es decir, existió esa libertad en principio infinita desde el primer hombre. Por eso Ortega afirma que «el hombre empieza cuando empieza la técnica. La holgura, menor o mayor, que ésta le abre en la naturaleza es el alvéolo donde puede alojar su excéntrico ser" (t. 5, p. 342). El hombre, entonces, es inventor $a b$ initio, $y$ su destino es accptar esa verdad y entregarse a la tarea de transformar, en la medida en que sea posible, su situación. Su destino es crearse un mundo donde pueda conquistar una felicidad relativa, donde pueda, cn fin, crear cultura. Aqứ cntra la técnica, que es nada menos que nuestra reacción metafísica a una situación igualmente metafísica, nuestra reacción humana a la vida como problema.

Compárese esto con la visión de Heidegger, en la cual el hombre era en la época pre-socrática una entidad apenas fragmentada o diferenciada, un ser pretecnológico que aún no abusaba de su poder para inventar $y$ alejarse del Ser. $Y$ el destino de ese hombre no sufre cambio alguno; en el siglo $\mathrm{Xx}$, en el apogeo de la modernidad, sigue siendo igual que antes: volver a ser un ente que deje que las cosas sean, que las respete tal como son y no las transforme para nada. Su destino, en fin, es volver a ser un ente que existe sólo para que el Ser -con mayúscula - sea proyectado dentro de la historia. Ni Borgman ni Heim, en su papel de filósofos de la técnica, han podido ir más allá de esta postura fundamental. A pesar de las intuiciones valiosas que han elaborado con respecto a la implicación de la técnica en la vida diaria y al problema de nuestra interacción con los ordenadores, en realidad ni el uno ni el otro han progresado más allá de una ontología heideggeriana a una visión más auténticamente "histórica" de la técnica. Y con esto, creo yo, palpamos los límites filosóficos de sus empresas.

Don Ihde, por otra parte, después de partir de un contexto heideggeriano, ha sabido distanciarse más que sus compatriotas en lo que, para nosotros, es lo más esencial aquí. Es verdad que Ihde concibe su reformulación sistemática de la técnica a la sombra del alemán: plantea una serie de "preguntas" que tienen una dimensión claramente ontológica, critica el platonismo que tiende a subordinar el aspecto materialista de la técnica a una abstracción conceptual que no comprende nuestra implicación existencial en ella, y reconoce a Heidegger como el creador de la filosofía de la técnica -l creador dice, no uno de los creadores-. Además, su deuda es, sobre todo, al primer Heidegger, cuyo análisis del "equipo" en Ser y tiempo o por lo menos una parte de él, evita la nostalgia que hemos apuntado arriba. ${ }^{10}$

Al mismo tiempo, sin embargo, Ihde ha visto algunos de los graves problemas que caracterizan la visión del Heidegger tardío. Ha leído el libro excelente de Michael Zimmerman titulado La confrontación de Heidegger con la Modenidad: la técnica, la politica, el arte (1990) y ha tomado en serio la descripción del maestro allí como un amodernista reaccionario" es decir, un pensador que, como muchos en su lugar y momento históricos, trató de resolver el problema de la modernidad desde una base filosófica marcadamente conservadora $y$ antimoderna." Como muestra Zimmerman con mucho detalle, esta actitud no sólo metafísica sino a la vez profundamente política y estética, le condujo a Heidegger, como a muchos otros en su querida Alemania, a aceptar, a su modo, el nacionalsocialismo creyendo que la salvación de esos 
ideales dependia de medidas drásticas. Este estudio de un especialista que antes publicó otro libro sobre Heidegger y simpatizaba mucho con su orientación filosófica, hace que el lector, cuando termina, se pregunte cómo puede uno seguir siendo un discípulo de Heidegger. En todo caso, Ihde no sólo ha leído este libro, sino que lo ha ponderado y ha repensado su propia actitud ante Heidegger. Y así en Technology and the Lifeworld es evidente un esfuerzo deliberado por parte de Thde de distanciarse de lo que llama «el romanticismo" de Heidegger. En un momento, por ejemplo, concluye:

Rechazo la idea que se ha popularizado a causa de Heidegger de que "sólo puede salvarnos un dios». Ni tampoco tengo fe alguna de que esto pudiera pasar. Desde una conciencia más aguda de nuestra contingencia, creo que más que nunca, tenemos que atender a nuestro propio destino en el acto de cuidar con diligencia y hasta con esmero de la textura tecnologica de nuestro mundo. Aunque no podemos simplemente ccontrolarlow - puesto que la cuestión está mal planteada así-, es verdad que podemos seguir ciertas direcciones y dar unos codazos significativos en los intersticios decisivos que existen [p. 163].

Las ideas expresadas en este pasaje son compatibles con la orientación historicista de Ortega. Primero, Thde rechaza enérgicamente una nostalgia que puede convertirse, como la historia ha mostrado, en una especie de fatalismo masoquista $-\mathrm{y}$ esto es la verdad para políticos, artistas, masas y filósofos-; segundo, Ihde intenta imaginarse una relación con la técnica que cuestiona la noción convencional del control y asi, de un salto, comienza a coupar un terreno que ya no es moderno; y tercero, él afirma y comienza a elaborar una relación con la técnica que quiere respetar nuestra radical contingencia. Ihde, en fin, adopta una postura más realista $y$, por consiguiente, parece que está dejando a un lado su afiliación heidcggeriana. Todo esto es perfectamente consecuente con el esfuerzo que hace en la ultima parte de Technology and the Lifeworld por encontrar un difícil equilibrio entre el pesimismo por un lado y el utopismo tecnocrático por otro. Asi, cuando Ihde insiste que slas tecnologías inclinan pero no determinan" (p. 183), cuando se dirige al carácter pluricultural y multiestable de la civilización transicional en que nos encontramos hoy - lo que nosotros llamamos la transición a la posmodernidad - y cuando él reconoce que tendremos que inventar soluciones para la crisis de la técnica dentro de los límites y posibilidades de este mundo, se mueve en una dirección que podría coincidir con la filosofia de la razón histórica de Ontega.

Porque para Ortega sólo podemos salvarnos de las crisis, sólo nos es posible salir a flote después del naufragio - y estamos naufragando en una modernidad cada vez más moribunda-, si nuestro punto de partida es una comprensión realista de quiênes somos y de exactamente cuál es nuestra situación actual. Nuestra experiencia de la vida, la conciencia de nuestro pasado y sobre todo de nuestros fracasos, es capaz de producir un sistema de conocimientos humanos que a su vez ureobra y se potencia en la historia como cognitio rerum gestarums (t. 6, p. 43). Esto es lo que Ortega llama en Historia como sistema da nueva revelación» que tanto necesita nuestro tiempo. Es una alternativa que queda por descubrir entre los filósofos de la tecnica en los Estados Unidos. Ahora bien, el proyecto de Ihde, como señalamos antes, queda incompleto. Ha escrito varios libros y ha progresado bastante en su comprensión de la técnica. Parece evidente que él está en una etapa de su propio pensamiento en la que intuye la necesidad de una base mctafísica más sólida y más rica que la que ha encontrado hasta la fecha, $Y$ parece también que el mismo está tratando de inventar esa base. Por mi parte, confieso que no creo que sea necesario volver 
a inventar la nueda. Sólo es necesario descubrir la filosolía de la técnica de Ortega y Gasset.

Para que tengamos un ejemplo de cómo el conocimiento de la obra de Ortega podría contribuir algo a la filosofia de la técnica en mi país, veamos otra vez una de esas frases que cité de Ihde arriba. Él dijo que hoy "más que nunca tenemos que atender a nuestio propio destino en el acto de cuidar con diligencia y hasta con esmero de la textura tecnológica de nuestro mundo». En inglés: "we must more than ever see to our own fate, by deeply an even caringly looking after our technologically textured world" (p. 163). Muy bicn y muy bien dicho. Pero cuando yo traduje esta frase la primera vez, iba a poner, en vez de "con diligencia y con esmero", simplemente aamorosamente». Thde no dijo esto exactamente, y por eso no lo traduje así. Pero cuando nos acercamos al problema que él trata de comprender desde la perspectiva de Ortega, es muy fácil dar ese paso y sentimos invitados... a amar nuestra téctica. Al fin y al cabo, para Ortega "yo soy yo y mi circunstancia», y esto significa que la técnica moderna no es nada secundaria sino que es una parte íntima de lo que somos, y que mientras neguemos esa dimensión o añoremos una vida sin la tensión que esa parte de nosotros produce, no va a ser posible convertir nuestra situación contemporánea -que es nuestro ser - en nada positivo. Querámoslo o no, nosotros, los hombres de fines del siglo $\mathrm{xx}$, somos una mezcla a la vez fabulosa y espantosa de la naturaleza y el artificio humano. Somos cuerpos materiales, arrojados aquí sin nuestra intervención, y al mismo liempo $-\mathrm{y}$ aparentemente sin que lo podamos alterar-, somos productos de la cultura, de nuestra poderosa capacidad para inventar. Somos también, como la máquina más sencilla, invenciones hurnanas. Si no aceptamos las consecuencias de esto, si no aprendemos a amar, en el mejor sentido, lo artificial, entonces nada va a cambiar y nuestra historia será una repetición de lo que siempre ha sido. Y yo creo que Ortega, Idhe, y todos nosotros queremos algo mejor.
1. Obras completas, tomo 5, Madrid, Revista de Occidente, 1983, pp. 223-224. Otras referencias a esta obra aparecerán entre paréntesis en d texto con el número del tomo seguido por las páginas.

2. Véase la excelente introducción de Carl Mitcham, ¿Qué es ia flosofia de la tecnología?, Barcelona, Anthropos, 1989.

3. Chicago / Londres, University of Chicago, 1984.

4. New Haven/Londres, Yale University, 1987.

5. Bloomington, Indiana University, 1990.

6. Vease Mark Okrent, Heidegger's Pragmatism: Understanding, Being, and the Critigue of Metaphysics, Ithaca / London, Cornell University Press. 1988 y Richard Rorty. Essays on Heidegger and Others: Philosophical Papers, vol. 2, Cambridge, Cambridge University Press, 1991.

7. En Thomas Sheehan (ed.), Heidegger: The Man and the Thinker, Chicago, Precedent, 1984, p. 57.

8. "Artificial Realities: Centering One's Life in an Advanced Technological Setting*, aparecerá en Fee- ling In Thought, redactado por Bernard Den Ouden y Marcia Moen, Nueva York, Peter Lang.

9. Lo dice en una carta personal y lo repite en su *respuestan a criticas de Stanley Carpenter y Manfred Stanley en "A Symposium on Albert's Borgman's Technology and the Character of Contemporary Life». Véase el tomo 4 de Philosophy and Tecimology, intitulado Technology and Contentporary Life y redactado por Paul $T$. Durbin, Dordrecht, Reidel, 1988.

10. Véase el excelente estudio de Hubert I. Dreyfus, "Between Techne and Technology: The Ambiguous Place of Equipment in Being and Time», en Michael Zimmerman (ed), The Thought of Martin Heidegger, vol. XXXII, Tulane Studies in Philosophy, 1984 .

11. Bloomington, Indiana University, 1990. También hay que mencionar el libro de Jeffrey Herf, Reactionary Modemism: Technology, Culture, and Politics in Weinar and the Third Reich. Nueva York, Cambridge University Press, 1984, cuya influencia Zimmerman reconoce. 